\title{
Singular spectrum analysis as a tool for early detection of centrifugal compressor flow instability
}

\author{
Alasdair Logan ${ }^{\mathrm{a}}$, David García Cava ${ }^{\mathrm{b}, *}$, Grzegorz Liśkiewicz ${ }^{\mathrm{c}}$ \\ ${ }^{a}$ Mechanical and Aerospace Engineering, University of Strathclyde, United Kingdom \\ ${ }^{b}$ Institute for Infrastructure and Environment, School of Engineering, University of Edinburgh, United Kingdom \\ ${ }^{c}$ Institute of Turbomachinery, Lodz University of Technology, Poland
}

\begin{abstract}
Centrifugal compressor machinery is subject to a potentially damaging phenomenon called surge at low mass flow rates. This effect may be preceded by a phenomena known as inlet recirculation - a flow reversal upstream of the impeller. A methodology to isolate inlet recirculation as a characteristic feature for monitoring of centrifugal compressor instability is presented in this study. The methodology is based on a nonparametric time series analysis technique called as singular spectrum analysis (SSA). SSA decomposes a signal into a number of Reconstructive Components (RCs), from which data trends and oscillatory components may be extracted. The frequency spectra of each RC and their relative contributions to the reconstruction of the original signal were examined and comparisons were made with spectral maps in existing literature. Individual and independent RCs were chosen to construct a compressor's instability monitoring system. Additionally, the performance of SSA was determined by the Window length parameter. The effect of modification of this parameter was also studied, and the various viable choices of component for the basis of inlet recirculation diagnosis were considered. The methodology was implemented in pressure dynamical signals measured in an experimental centrifugal compressor rig. High frequency pressure measurements were taken at a number of flow conditions and locations within the compressor. The results demonstrated the potential of a methodology based on SSA to identify and extract oscillatory components with information about the local effect of inlet recirculation and eventually successfully monitor centrifugal compressor's instability. Keywords: Singular Spectrum Analysis, Inlet Recirculation, Surge, Centrifugal Compressors, Condition Monitoring, Flow instability
\end{abstract}

\section{Introduction}

Centrifugal compressors are susceptible to unstable phenomena at low mass flow rates [1. Surge, as discussed by Gravdahl and Egeland, is the most dangerous of these effects [2. Rotating stall is also known to occur in centrifugal impellers, but its character, according to De Jager [3] is more local. Surge was first studied by Emmons [4, a mathematical model of surge in axial compressors was developed by Greitzer

\footnotetext{
${ }^{*}$ Corresponding author

Email address: david.garcia@ed.ac.uk (David García Cava)
} 
[5] and was successfully applied to centrifugal impellers by Hansen [6]. The Greitzer model includes the compressing system consisting of the compressor (simulated by means of the flow channel), outlet network (simulated by means of the large pressure vessel named as the plenum), and the outlet valve (simulated by means of the flow channel). The inlet piping was not included in the original model as the surge develops in the plenum [5]. The experimental studies of the influence of the inlet network have indeed shown that the inlet piping has much smaller importance. It does, however, slightly affect the oscillation amplitude [7] and the surge line position 8 .

Compressor pressure ratio reaches a maximum near and within the so-called surge margin. The machine, however, must not operate in this area in order to avoid potentially damaging severe flow oscillation [2]. Botros and Henderson went on to survey various proposed anti-surge systems and divided them into three groups 9]. Classic anti-surge systems impose limited operation range based on the surge margin to keep the machine away from this dangerous condition. This induces a significant limit to the operating range. Moreover, the limit itself is often based on theoretical estimation [10] or difficult experimental techniques 11. The active anti-surge methods often deployed mechanical systems, such as valves or movable vanes, to suppress the surge [12, 13]. According to Gravdahl [14, one of the promising solutions involved a closecoupled valve as was proposed by Simon et al [15]. These systems allow the machine to operate in the region which is not available with passive techniques. This, however, comes with a price of additional necessary infrastructure that needs to be configured and maintained. The investigations outlined in this work aimed to identify a system that could supply a control system with information indicative of proximity to surge by means of identifying instabilities that might occur before surge. Botros and Henderson [9] named this class of protection schemes as protection and control anti-surge system. Systems of this kind could give a good ratio between large operating infrastructure and costs (active systems) and substantial losses in the operating range (passive systems).

'Protection and Control' anti-surge systems are as good as their reliability in identifying proximity to surge. This information might come from local unstable flow structures that appear prior to the surge. Inlet recirculation was proven to be efficient in this role [16]. Instability of this kind has been also detected in centrifugal compressors prior to more severe phenomena such as the surge or the rotating stall [17, 18. According to previous studies, the inlet recirculation has a very distinctive pressure signature. It generates a region of increased average static pressure with its short and chaotic drops. The frequency domain analysis does not reveal any dominating frequencies. The phenomenon induced noise in wide frequency spectrum [16] and increment of the average static pressure in the recirculation zone [19]. Numerical study of this phenomenon allowed to form hypothesis making connection between the braodband noise and the inlet recirculation: the radial thickess of the recirculation zone is varying in time in a non-periodic manner. When it's thin enough, the high pressure zone disappears [19] and the pressure drop is observed [20. Due to its very particular features, the inlet recirculation is already used in different configurations of the anti-surge system [21, 22]. 
One of the most important aspects of monitoring and controlling a dynamical system is to select characteristic features that are sensitive to any changes of the defined normal conditions. Therefore, selecting features that could be used to alert, in advance, the presence of instability are of interest for real-time monitoring of centrifugal compressors. Then, techniques that are able to separate and and eventually isolate particular features from dynamical signals of physical quantities will potentially support reliable and robust condition monitoring systems. Investigating the changes in the dynamic pressure measurements has demonstrated good results in detecting surge onset in compressing machines [23. This was done by gradually decreasing the mass flow rate to identify any change in the measured dynamical signals. The measured signals are generally noise and this can mask the characteristic features, therefore some signal processing techniques might be conducted. For instance, Bulot et al. applied low-pass filter or [24] Lawless et al. [25] a band-pass filter. These techniques are successful but they require a prior information, for instance knowledge about the particular frequency range of interest. Alternatively, there have been other studies where a fixed throttling valve was set to analyse the frequency spectrum at particular working condition. This allow the study on the performance of the compressing machine by comparing the different frequency spectres at different working conditions as shown in [26. The analysis demonstrated the complexity of unstable flow structures on the frequency spectra and the need for tools to alleviate this information and easing the interpretation of the instabilities. Other approaches that have been implemented are the use of time-frequency analysis such as continuous wavelet transform (CWT) as presented in [27] where the identification of rotating stall was found to start near the impeller leading edge. The CWT has been used in the same compressing unit used in this work for analysing the surge onset [28]. The result demonstrated that local instabilities were successfully detected in the range of $300-1000 \mathrm{~Hz}$ namely inlet recirculation presented as a broadband noise and non observable periodic peaks as also was found in [29, 23]. The results of the CWT depends on the selection of the mother wavelet as well as the resolution in time and frequency domain. [30. The surge peak was clearly noticeable with this method, but early flow instabilities proceeding surge were resulting in a weak peak of not-constant amplitude. Recently the use of Empirical Mode Decomposition (EMD) [31] has been investigated in order to decompose the measured dynamical signal in independent components and hence investigate the presence of instabilities in each intrinsic mode.

Methodologies based on singular value decomposition (SVD) [32, 33. have demonstrated to be good candidates for these applications. It is, therefore, worth inspecting whether this methods can extract the features of the inlet recirculation. Singular Spectrum Analysis (SSA) is a nonparametric modification of the Principal Component Analysis for non-independent variables such as time series. The basics of SSA is to decompose time series by means of trend and different oscillatory components. The caterpillar method of SSA was used, as described by Golyandina et al [34]. This version of SSA emphasized the separability of the various Reconstructed Components (RCs) generated [35.

Initial uses of SSA were climatological and sociological. Trends were extracted from time series of weather parameters by Ghil et al [36. SSA has been also been compared with CWT and EMD in multiannual time 
series in coastal transitional waters. The study compares the advantages and limitations of each method. The study concludes that SSA performs the best as it adds also good feature extraction when the measured signals are incomplete. However, it is also discussed the value of combining the methods depending on the nature of the analysis as each method could complement to each other [37. Mechanical applications of SSA have examined periodic vibration in complex dynamical systems [38] and a modified version to be applied in the frequency spectrum responses for structural health monitoring of wind turbine blades [39].

The resolution of the signal decomposition performed by SSA, expressed by the number of components obtained, was governed by the so-called Window Length. Leles at al expressed the effect of modification of this parameter as separability [40]. The study presented in this manuscript is a continuation of work carried out by Garcia et al, where SSA was used to study static pressure measurements from a centrifugal compressor experiment [41, 42]. Garcia et al noted that the the total pressure ratio across the compressor dropped in the region of inlet recirculation, it was thought that identification and avoidance of this phenomenon would allow for optimal operation of the studied compressor. Therefore, SSA is a good candidate for this type of analysis as it is important to isolate independent components that correspond to identified characteristics for better understanding of the performance of the compressing unit. This work has proven the viability of condition monitoring based on SSA, through identification of the contribution of each obtained component to the reconstruction of the signal. It was hypothesised that inlet recirculation could be isolated and extracted from the original signal by the use of SSA.

Therefore with this study is attempted to use inlet recirculation as an indicator of the surge inception which was already observed in this experimental rig [16, 28. These studies identified a particular region in the frequency spectra that increases in amplitude when inlet recirculation appears. This phenomenon is analysed with the mentioned non-parametric method, Singular Spectrum Analysis. The aim of study is to analyze whether SSA can be used for extraction of the signal associated with the inlet recirculation and hence use this portion of the signal as a potential characteristic feature for condition monitoring. The study also aims to provide a wider analysis of the factors influencing the SSA methodology in the application to the pressure signals.

To this end, the work presented in the manuscript is organised as follows: first a methodology is presented to investigate the capabilities of SSA for monitoring centrifugal compressor's instability. The experimental set up with a description of the test rig and the data acquisition campaign is presented. The results and discussion section is divided in three main sections: (i) The study of the information included in each independent reconstructive components. (ii) The study of pressure spectral maps to identify the inlet recirculation phenomena at different working conditions. (iii) The study of the methodology performance for different parameters. Finally the conclusions of the study are presented to define the limitations of the method and future research ideas. 


\section{Methodology}

The methodology proposed for decomposing pressure dynamical signals measured in a centrifugal compressor into a set of independent oscillatory components is based on Singular Spectrum Analysis technique. This permits to isolate and/or reconstruct some components of the original signal for better understanding of the phenomena and for extracting characteristic features for successful condition monitoring of centrifugal compressors. The main steps of the conventional SSA are embedding, decomposition and reconstruction (grouping and diagonal averaging) are presented in the following sections. SSA was performed according to the technique prescribed by [34, 43].

\subsection{Data collection and embedding}

A dynamical pressure signal is measured and discretised into a vector $\mathbf{x}=\left(x_{1}, x_{2}, \ldots, x_{N}\right) \in \mathbb{R}^{N}$ where $N$ is the length of the vector signal. A trajectory matrix is constructed through horizontal concatenation of a series of lagged vectors. These lagged vectors were derived from the original vector signal, $\mathbf{x}$ according to the parameter $L$, window length defined as $1<L \leq N / 2$. Thereby, a trajectory matrix $\mathbf{X}$ is constructed with a Hankel form and dimension $[L \times K]$ where $K=N-L+1$.

$$
\mathbf{X}=\left[\begin{array}{ccccc}
x_{1} & x_{2} & x_{3} & \cdots & x_{K} \\
x_{2} & x_{3} & x_{4} & \cdots & x_{K+1} \\
x_{3} & x_{4} & x_{5} & \cdots & x_{K+2} \\
\vdots & \vdots & \vdots & \ddots & \vdots \\
x_{L} & x_{L+1} & x_{L+2} & \cdots & x_{N}
\end{array}\right]
$$

\subsection{Decomposition}

The next step is the decomposition of the trajectory matrix $\mathbf{X}$. An equivalent solutions is obtained with the eigenvalue decomposition of the squared matrix $\mathbf{S}=\mathbf{X} \mathbf{X}^{t}$ with dimension $[L \times L]$ where $[\cdot]^{t}$ represents transpose. Therefore, this decomposition achieves a set of eigenvalues in decreasing order $\left(\lambda_{1} \leq \lambda_{2} \leq\right.$ $\left.\cdots \leq \lambda_{L}\right)$ and their corresponding eigenvectors $\left(\mathbf{u}_{1}, \mathbf{u}_{2}, \ldots \mathbf{u}_{L}\right)$ of $\mathbf{S}$. Thus, the decomposition of $\mathbf{S}$ leads to individual components where the sum of all of them results in the original trajectory matrix $\mathbf{X}$ defined in Eq. (1) as $\mathbf{X}=\mathbf{X}_{1}+\mathbf{X}_{2}+\cdots+\mathbf{X}_{i}+\cdots+\mathbf{X}_{L}$. Each individual component is defined as

$$
\mathbf{X}_{i}=\sqrt{\lambda_{i}} \mathbf{u}_{i} \mathbf{v}_{i}^{t}
$$

where

$$
\mathbf{v}_{i}=\frac{\mathbf{X}^{t} \mathbf{u}_{i}}{\sqrt{\lambda_{i}}}
$$

As seen in Eq.22, each individual matrix component $\mathbf{X}_{i}$ is defined by its corresponding eigentriple set $\left(\sqrt{\lambda_{i}}, \mathbf{u}_{i}, \mathbf{v}_{i}\right)$ of the singular vector decomposition of $\mathbf{X}$. Finally, the Principal Components (PCs) vectors 
are obtained by projecting the trajectory matrix onto the eigenvectors as follows

$$
\mathbf{p}_{i}=\mathbf{X}^{t} \mathbf{u}_{i}=\sqrt{\lambda_{i}} \mathbf{v}_{i}
$$

130

\subsection{Reconstruction}

Each individual component matrix $\mathbf{X}_{i}$ contains particular information of the original trajectory matrix $\mathbf{X}$ and hence each one contributes more or less towards the reconstruction of $\mathbf{X}$. Additionally, it can be added that each $\mathbf{X}_{i}$ correspond to its own eigentriple. As the eigenvalues $\lambda_{i}$ are in decreasing order, the first individual component matrices contributes more than the last individual component matrices. These individual component matrices can be grouped to reconstruct a portion of the trajectory matrix but they can be also used individually to investigate the information included in each individual component matrix.

In order to reconstruct each individual component, it is needed to convert the individual component matrices by diagonal averaging. Let $y_{m, n-m+1}$ be an element of an individual component matrix $\mathbf{X}_{i}$. Then, the Reconstructive Components (RC) corresponding to this individual component matrix $\mathbf{X}_{i}$ are calculated as follows

$$
r_{i n}= \begin{cases}\frac{1}{n} \sum_{m=1}^{n} y_{m, k-m+1} & 1 \leq n \leq L \\ \frac{1}{L} \sum_{m=1}^{L} y_{m, k-m+1} & L \leq n<K \\ \frac{1}{N-n+1} \sum_{m=n-K+1}^{L} y_{m, k-m+1} & K \leq n<N\end{cases}
$$

To this end, $L$ independent RCs are obtained by the decomposition of a vector signal $\mathbf{x}$. Therefore, the original vector signal $\mathbf{x}=\left(x_{1}, x_{2}, \ldots, x_{N}\right) \in \mathbb{R}^{N}$ is now decomposed into a set of reconstructive components $\Gamma_{x}$ for independent examination as shown in Eq. 6

$$
\mathbf{x}=\Gamma_{x}=\mathbf{r}_{1}+\mathbf{r}_{2}+\cdots+\mathbf{r}_{L}=\sum_{i=1}^{L} \mathbf{r}_{i}
$$

\subsection{Feature Extraction}

The nature of the decomposition and the relationship between each component with physical flow features was of interest, therefore all $L$ RCs were independently examined. It was hypothesised that an individual RC could be associated with inlet recirculation. An optimised SSA process would only subject this identified RC to diagonal averaging, but in this investigation all PCs were converted to RCs and studied. The nature of each RC changed with $L$ and this effect on the potential outcomes of the SSA decomposition was considered in detail in section 4.3

As mentioned in the introduction, inlet recirculation is a phenomenon that may occur immediately prior to surge. Therefore, monitoring only the RC (or RCs) that contains the oscillations of the inlet recirculation will enhance a continuous monitoring of this phenomena. Thus, the measured vector signal will be cleaner and 
only relevant information will be retained. This will give an indication of compressor's instability proximity

\section{Test rig}

This study was conducted on the low-speed single stage centrifugal blower DP1.12. Figure 1 presents the cross-section of the blower and position of two considered measuring points. Descriptions in the figure correspond to: A - inlet pipe, B - Witoszynski nozzle, C - impeller, D - vaneless diffuser, E - volute, F an asynchronous AC motor with the rotational speed $f_{\text {rot }}=100 \mathrm{~Hz}$. The nominal flow rate was equal to $\dot{m}_{n}=0.75 \frac{\mathrm{kg}}{\mathrm{s}}$, where the pressure ratio was equal to $\pi=1.08$, the dimensionless mass flow rate coefficient $\phi=1.4$ and $T O A_{n}=32 \%$. The choice of the test stand was influenced by two factors. Firstly, this low-speed blower is safe to operate in the surge for certain time. Secondly, this setting is well examined [16] hence the results of current analysis could be easily associated with the known machine conditions. Figure 2 presents the performance curve of the blower. Four measurement points were affected by the back flow coming from the inlet recirculation (marked with dark-blue points). This affected the mass-flow measurement accuracy, hence those points are displayed for information only. The performance curve fit was made based on the square data points.

Now the RMS values of $\mathbf{R}_{\mathrm{I}}$ can be used as metric for monitoring centrifugal compressor instability. Relative large values of RMS are present in pressure signals with large amplitude and hence they are closer to unstable working conditions. On the other hand, relative small values of RMS are obtained in measured pressure signals working in stable conditions.

To this end, a methodology to monitor the instability of centrifugal compressors based on inlet recirculation is defined and summarised in the following steps:

1. Measure a pressure dynamical signal and discretised it in a vector signal.

2. Decompose the vector signal into a set of RCs.

3. Identify the RC/RCs that might have information about the inlet recirculation and compute its RMS.

4. Use the relative RMS's values to monitor the working conditions of the centrifugal compressor.

- outlet pipe. The most important stand dimensions are listed in the table 1 The rotor was driven by $h_{n}=0.75 \mathrm{~s}$, where the pressire ratio was equal to $\pi=1.08$, the dimensionless mass flow rate coeficient 
Table 1: Table of most important dimensions of the test rig

\begin{tabular}{lcc}
\hline Parameter & Notation & Value \\
\hline Inlet pipe diameter & $D_{i n}$ & $300 \mathrm{~mm}$ \\
Rotor inlet diameter at hub & $D_{1 h}$ & $86.3 \mathrm{~mm}$ \\
Rotor inlet diameter at shroud & $D_{1 s}$ & $126 \mathrm{~mm}$ \\
Rotor inlet span & $b_{1}$ & $38.9 \mathrm{~mm}$ \\
Rotor outlet diameter & $D_{2}$ & $330 \mathrm{~mm}$ \\
Rotor outlet span & $b_{2}$ & $14.5 \mathrm{~mm}$ \\
Diffuser outlet diameter & $D_{3}$ & $476 \mathrm{~mm}$ \\
Rotational frequency & $f_{\text {rot }}$ & $100 \mathrm{~Hz}$ \\
Impeller tip speed & $u_{2}$ & $103 \frac{\mathrm{m}}{\mathrm{s}}$ \\
Number of blades & $z$ & 23 \\
Blade Passing frequency & $f_{B P}$ & $2.3 \mathrm{kHz}$ \\
Valve position at nominal conditions & $T O A_{n}$ & $32 \%$ \\
\hline
\end{tabular}

Table 2: Working regimes identified in this compressor.

\begin{tabular}{llll}
\hline TOA & Stability & Gauges & Name \\
\hline TOA $>27 \%$ & stable & all & nominal work \\
TOA $\in(17 \%, 27 \%)$ & locally unstable & inlet & inlet recirculation \\
TOA $\in(10 \%, 17 \%)$ & unstable & all & transient phase \\
$t<10 \%$ & unstable & all & deep surge \\
\hline
\end{tabular}

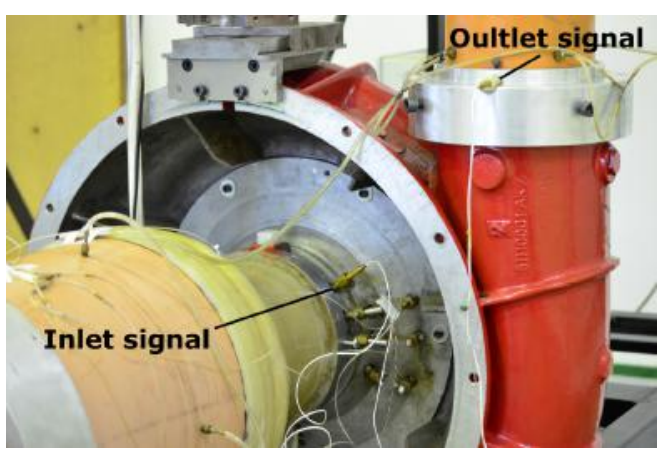

(a)

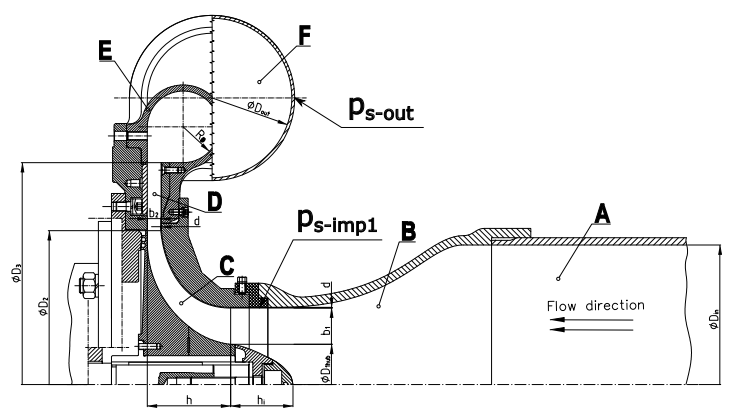

(b)

Figure 1: Experimental rig. (a) Picture of the experimental rig. (b) Cross-section of experimental rig with relevant pressure transducer locations and dimensions. 41

\subsection{Data collection procedure}

Subminiature Kulite transducers were used to measure static and total pressures of the flow at various points. Figure 1 shows the arrangement of these transducers. Sensor $\mathrm{p}_{\mathrm{s}-\mathrm{imp} 1}$ was of particular interest, as it was upstream of the impeller tip, where recirculatory flow was detected [16]. Outlet measurements, at $\mathrm{p}_{\mathrm{s}-\mathrm{out}}$, where the local phenomenon of inlet recirculation was absent, were used as the basis of a comparison. Each sensor gathered $2^{21}=2097152$ samples collected at a frequency of $100 \mathrm{kHz}$.

A throttling valve at the outlet allowed for flow rate control, which was quantified by the Throttle Opening 
Area (TOA), expressed as a percentage. Measurements were taken at 1 percent increments of TOA, with an adequate settling time to eliminate transient phenomena associated with throttling.

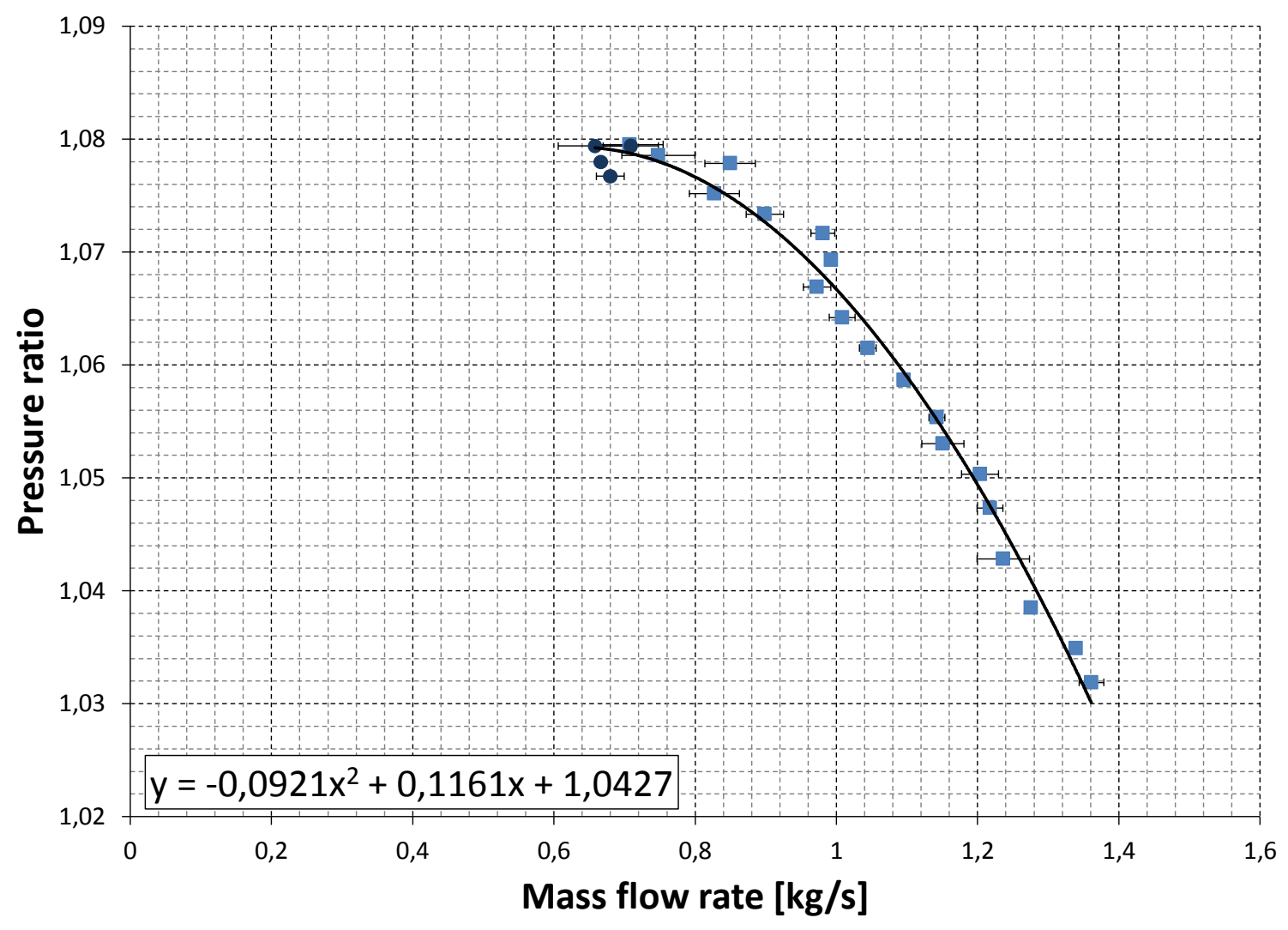

Figure 2: The performance curve of the centrifugal blower used in this study.

\section{Results and discussions}

\subsection{Frequency spectra of individual reconstructive components}

The frequency spectra of individual RCs were obtained by a Fourier transform. The rationale of this study examined the frequency domain behaviour of the various components obtained by the SSA decomposition. Parallel plots of a range of RCs were generated in order to elucidate the nature of the decomposition.

Initial investigations were performed with the aim of demonstrating the ability of SSA to break down a signal into a number of components. The behaviour of this process was to be studied by parallel plots of frequency spectra. A single throttle position, $\mathrm{TOA}=22 \%$, was chosen, and the pressure signals were measured in the sensor location, $\mathrm{p}_{\mathrm{s}-\mathrm{imp} 1}$. This was the operating point at which inlet recirculation was most prominent, and therefore was of greatest interest to this study. Sample window lengths were chosen such that the variable performance of SSA could be displayed at $L=10,50$ and 200 . The frequency spectra of 10 
reconstructed components were plotted in parallel, so as to ensure the clarity of the plots while capturing a sufficient portion of the full signal.

Fig. 3a shows the first of these parallel plots, with a window length of $L=10$. This plot shows how SSA decomposes the signal into a number of components equal to the window length - in this case a full decomposition is shown. Here the original signal could be reconstructed by the summation of all ten components. It was noted that the majority of the original frequency spectrum was contained within RC 1, this was a direct result of the process of eigenvector ordering described in section 2.2 Inlet recirculation is responsible for a broadband of oscillation at around $1000 \mathrm{~Hz}$ at $\mathrm{p}_{\mathrm{s} \text {-imp1 }}$ [16], most prominently at $\mathrm{TOA}=22 \%$. This phenomena can be clearly seen as a band of high frequencies in RC 1. This first component also contained the majority of lower frequency oscillations such as the impeller rotational frequency $f_{\text {rot }}=100 \mathrm{~Hz}$ and the blade passing frequency, $f_{\mathrm{BP}}=2.3 \mathrm{kHz}$. It is important to reflect that after the decomposition, each $\mathrm{RC}$ will contain different information about the original signal by means of dynamical variability content labelled by their corresponding eigenvalues. If the purpose of the analysis is to reconstruct the original signal with a certain level of approximation, then eigenvalues can be used to estimate the desired number of RCs for the reconstruction; as seen in a study considering the same compressor unit [41. On the other hand, if the analysis is to isolate a particular signature of the original signal as in this study, then it is important to interrogate the frequency spectral decomposition as shown in 3 and the spectral maps as discussed in section 4.2 .

The potential applicability of SSA at this window length was unclear - the discarding of RCs 2-10 would have a limited effect on the original signal, and any flow phenomena contained within these components would be of practically negligible importance. All flow features of interest were embedded within RC 1 SSA had limited ability to isolate particular phenomena. It was, therefore, concluded that selection of a higher window length would be required to optimally utilise SSA.

The window length was increased to $L=50$, as plotted in Fig. 3b 10 RCs were represented in the plot, for purposes of clarity and comparison, but it should be remembered that the original signal was now discomposed into a total of $50 \mathrm{RCs}$. This window length was used in previous studies [41, 42] because it corresponded to a frequency close to the blade passing frequency, over which there was no significant energy in the Fourier spectrum. Therefore this was an obvious choice for the next investigative step. By comparison of Figs. $3 \mathrm{a}$ and $3 \mathrm{~b}$, the effect of window length modification was clear. It was now seen that increasing the number of components into which the signal was discomposed resulted in a diminished fraction of the original spectrum captured in RC 1. This caused an increased apparent importance of RCs 2-10, as expressed by the range and magnitude of the frequencies contained therein. The increased importance of RCs 2 and 3 was of particular interest, with these components giving a clear representation of inlet recirculation. This effect is 
not entirely captured by either component, but it was envisaged how analysis of, for example, RC 2 could allow indication of flow instability. This informed subsequent attempts to demonstrate the variation in RC 2 with throttle position, as in section 4.2 and section 4.3 . The blade passing frequency $f_{\mathrm{BP}}$ was seen to shift to RC 3 at this new window length. Unlike inlet recirculation, responsible for a broad band of oscillation, it was postulated that this point phenomenon could be entirely isolated at a sufficiently high window length.

Finally, a window length of $L=200$ was chosen to investigate the effect on the decomposition behaviour, quadrupling the number of RCs and further decreasing the dominant stature of RC 1, at the expense of increased computational demand. Fig. 3c shows the effect of this inflated window length selection. RC 1 has diminished in importance to such a point where almost no oscillation indicative of recirculation is contained within. This component now exclusively captured the lowest, generally higher magnitude frequencies present in the original data. The relative significance of RCs 2-10 was seen to increase, although it should be noted that while the inlet recirculation has effectively been removed from RC 1, it was now spread across a wider range of components. $\mathrm{RC} 2$, promising at $L=50$, now captured phenomenon below the frequency at which the inlet recirculation was most prominent. The blade passing frequency, $f_{\mathrm{BP}}$, was shifted from $\mathrm{RC} 3$ to $\mathrm{RC}$ 10. These outcomes demonstrated how higher frequency components generally appeared in higher RCs with an increasing window length. It seemed that RC 9 would be a better indicator of the recirculation than RC 2 at $L=200$; this is further discussed in section 4.3 .

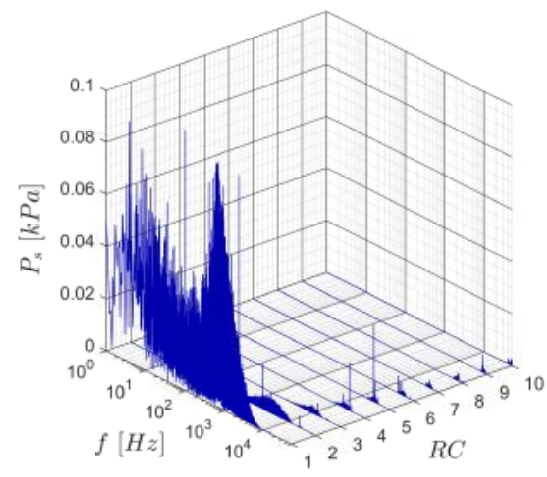

(a) $\mathrm{TOA}=22 \%, L=10$

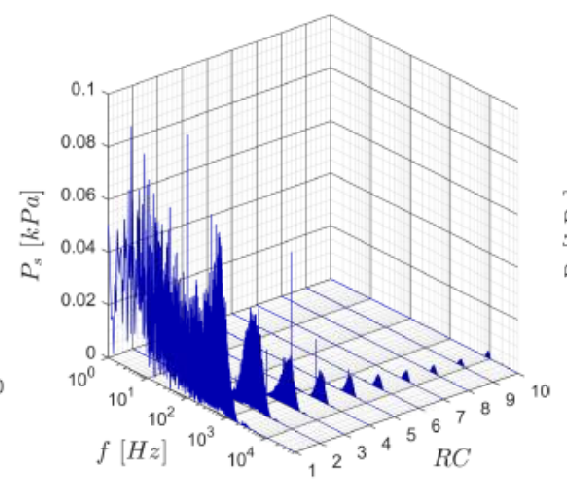

(b) $\mathrm{TOA}=22 \%, L=50$

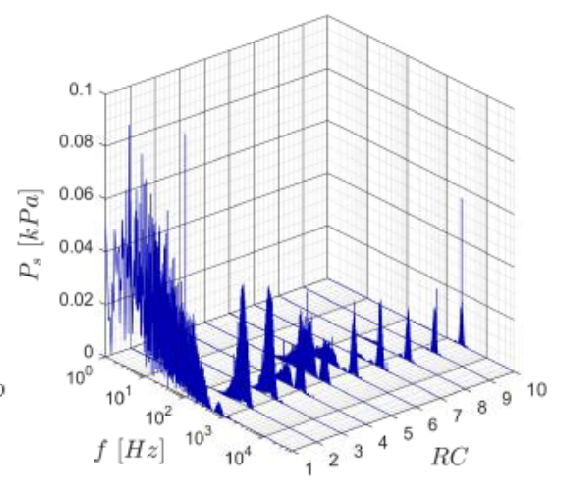

(c) $\mathrm{TOA}=22 \%, L=200$

Figure 3: Frequency Spectra of RCs 1-10 at the upstream of the impeller ( $\left.\mathrm{p}_{\mathrm{s}-\mathrm{imp} 1}\right)$ for different window lengths

\subsection{Pressure spectral maps}

It was now thought necessary to demonstrate the variation in behaviour of the decomposed components with throttle position. A method was derived from [16, where frequency spectra were represented at the full range of throttle positions in so-called spectral map. A window length of $L=50$ was chosen for these plots, since $L=10$ was ruled out in section 4.1 and $L=200$ was a large value of window length and it requires a significant computational demand unneeded for the demonstration of the purpose of this analysis. A logarithmic pressure amplitude scale and a sampling frequency of $100 \mathrm{kHz}$ was replicated in these plots in 
order to encourage a more valuable comparison process. Throttle position was limited from $5 \%<\mathrm{TOA}<40 \%$ in this study, where instability at low flow rates was of particular interest. The computational demand induced by the abundance of data plotted was tempered by limiting the signal length to 1 million samples such a limitation did not produce any divergence with the full signal plotted in [16].

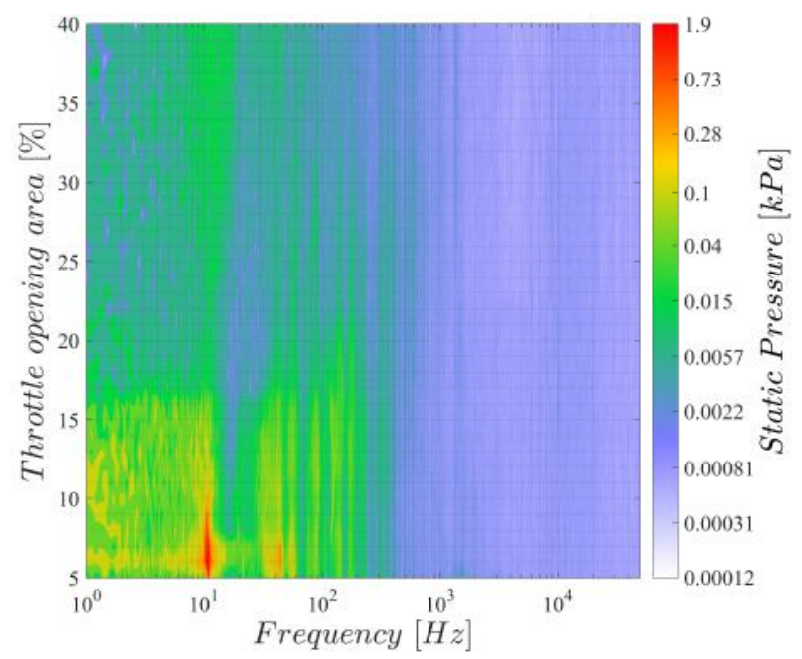

(a) $p_{\text {s-out }}$

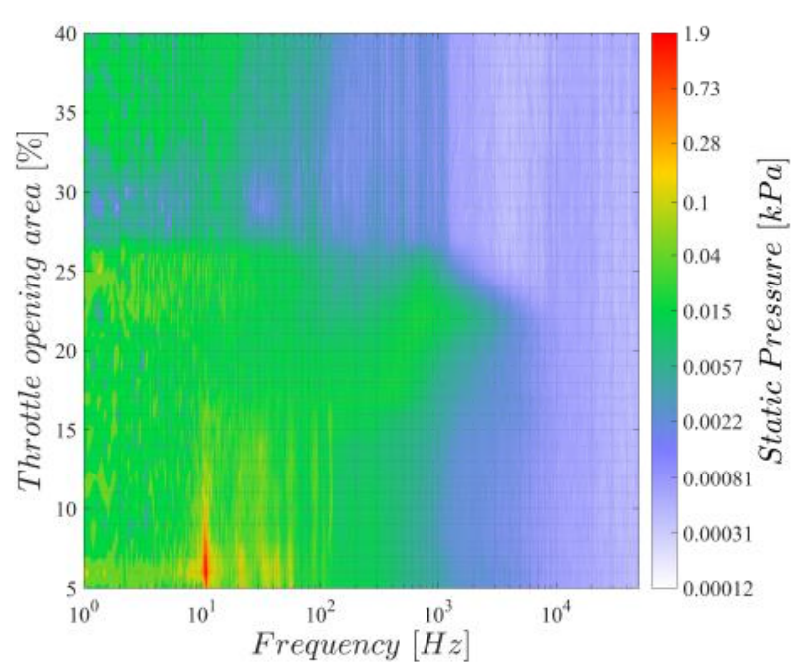

(b) $\mathrm{p}_{\mathrm{s}-\mathrm{imp} 1}$

Figure 4: Pressure spectral maps of the raw signals at the outlet and at the upstream of the impeller

RC 1 was plotted according to this method at both sensor locations, outlet $\mathrm{p}_{\mathrm{s} \text {-out }}$ and upstream of the impeller $\mathrm{p}_{\mathrm{s}-\mathrm{imp1}}$ as in Fig. 5. Outlet measurements, wherein the inlet recirculation was absent, were provided so as to convey the local character of the phenomenon around which this research was focused.

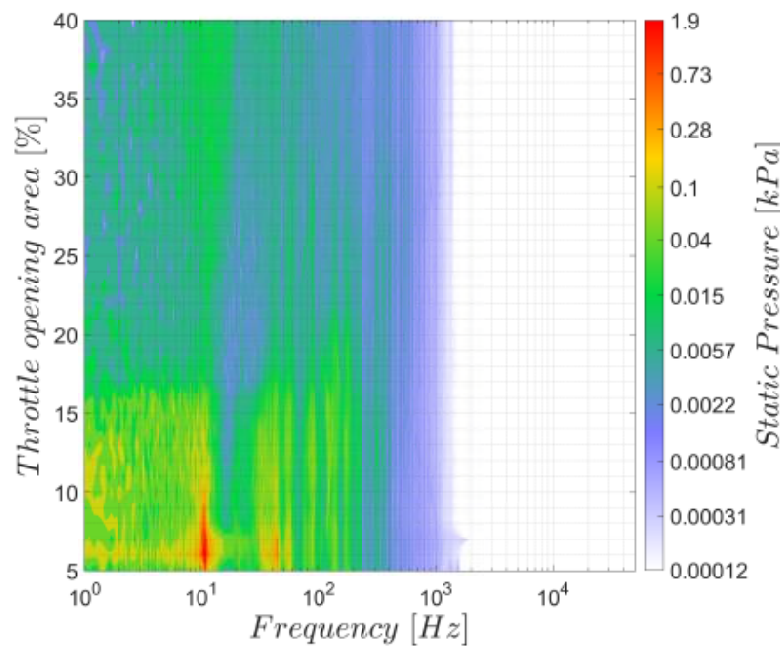

(a) $p_{\text {s-out }}$

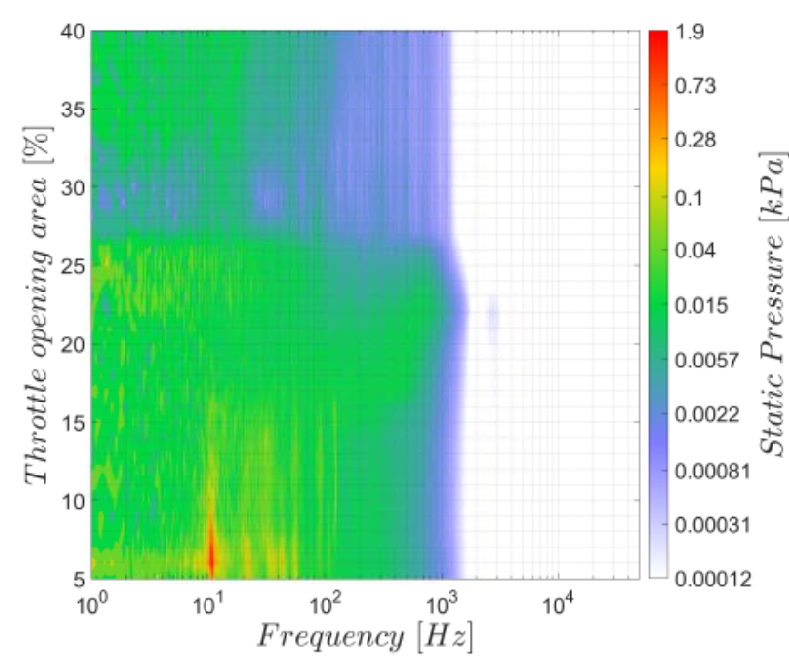

(b) $\mathrm{p}_{\mathrm{s}-\mathrm{imp} 1}$

Figure 5: Pressure spectral maps of RC 1, 5\% < TOA $<40 \%$ at the outlet and at the upstream of the impeller

It was previously established that $\mathrm{RC} 1$ contained the great majority of the data in the original signal. 
This was confirmed by comparison between figures 4 and 5 where the full signal can be compared to the relative contribution of RC 1 . The above figures were effectively identical within the first $1000 \mathrm{~Hz}$ of the original signal, with higher frequencies captured by components 2 and above, as was expected from analysis of Fig. 3. The appearance of the deep surge, manifest as a frequency peak at $10.8 \mathrm{~Hz}$ at lower TOAs was seen at both sensor locations this was representative of the global character of the deep surge. This frequency was approximately predicted by calculation of the Helmholtz frequency, $\mathrm{f}_{\mathrm{H}}=11.5 \mathrm{~Hz}$ which is a theoretical surge frequency [44] predicted by the Greitzer model [5], where the internal volume of the experimental compressor rig was treated as a Helmholtz resonator. This technique for predicting the resonance at deep surge was suggested in [10] and appeared to provide a reasonable approximation of the resonant frequency.

RC 2 offered a promising spectrum for the detection of the recirculation in Fig. 3b and it was thought that showing how this component developed with throttle position could provide useful insight into the function of SSA. This phenomenon was identified as an area of high frequency $(\sim 1000 \mathrm{~Hz})$ pressure oscillation at $17 \%<$ TOA $<27 \%$. It had been established from Fig. $3 \mathrm{~b}$ that the inlet recirculation was partially captured by RC 1 , and this was confirmed through inspection of Fig. $5 \mathrm{~b}$ No such region was seen at the outlet, where some indication of instability appeared at $\mathrm{TOA}=17 \%$ but this was attributed to the transient phase appearing prior to the surge. The clear peak of the deep surge can be noticed around $T O A=11 \%$. Comparison with Fig. 4 however, showed that the limitation of RC 1 to capture higher frequencies meant that much of the area identified as being indicative of the inlet recirculation was absent; it was thought this would be captured by RC 2, as shown in Fig. 6 .

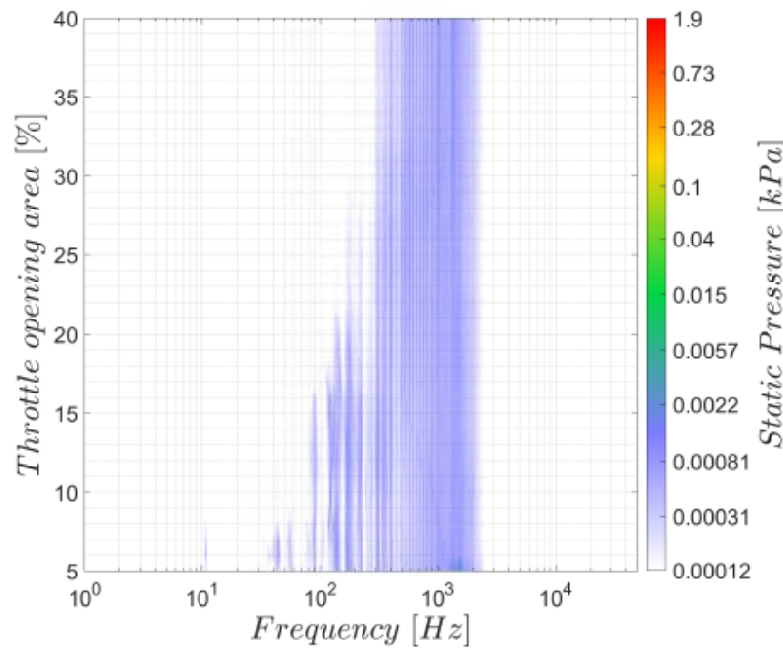

(a) $p_{\text {s-out }}$

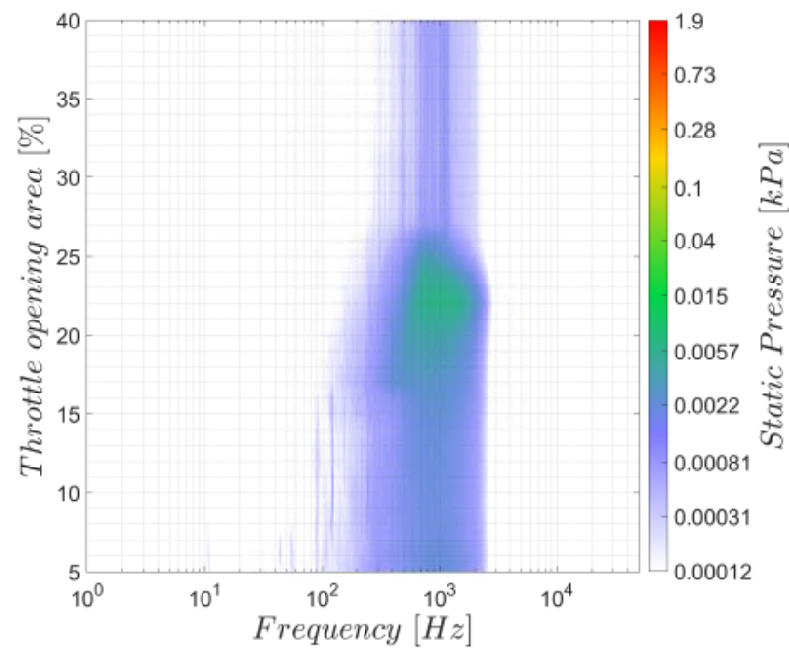

(b) $\mathrm{p}_{\mathrm{s}-\mathrm{imp} 1}$

Figure 6: Spectral maps of RC 2, 5\% $<\mathrm{TOA}<40 \%$ at the outlet and at the upstream of the impeller

Fig. 6 shows RC 2 subjected to the spectral map plotting method. The logarithmic colour bar allowed $\mathrm{RC} 2$ to be represented at the same scale as RC 1, but the great difference in magnitude should be noted. 
The appearance of the inlet recirculation at $\mathrm{TOA}=26 \%$ was clear in Fig. $6 \mathrm{~b}$ this was marginally later than was seen in RC 1. It could be suggested that RC 1 captured the inlet recirculation at a higher TOA due to the pressure oscillation at the onset of this feature occurring within the range of frequencies generally captured by RC 1 . As this feature increased in prominence it was manifest as a broader band in the frequency spectra and RC 2 was required to reproduce this oscillation. The indication of the inlet recirculation with $\mathrm{RC} 2$ is, however, much more clear as the phenomenon is isolated from the background. Again there was some instability appearing within the region of the transient phase at $\mathrm{p}_{s-\text { out }}$ as shown in Fig. 6a, but no indication of the inlet recirculation, as was expected.

\subsection{SSA-based methodology parameter selection and its effects}

\subsubsection{Effect of the Window length}

The selection of the window length is data and analysis dependent. In general, the rationale on the selection of the window length is to decompose the input data into a certain number of independent and interpretable reconstructive components. One method that has been proposed to select an appropriate window length is a statistical test based on auto-correlation coefficient and it has been used in several studies [45, 46, 47. The principal is to select a window length equal to the number of lags that define the correlation length when the auto-correlation coefficient is zero. This will set a certain number of reconstructive components, which can be considered to be linearly independent. A selection of a window length near any zero-crossing of the auto-correlation function will then be a potential candidate. The number of reconstructed components into which the input signal is decomposed is governed by the window length and depends on the desired type of analysis. In order to characterize how effectively flow features can be separated by this decomposition, the weighted correlation has been proposed as a metric [48. This is very useful when a partial reconstruction of the original signal is required and thus provides an indication of the number of required RCs. In this study, the intent is to isolate the effect of the inlet recirculation in a single and independent RC. As discussed in previous studies [23, 29, the inlet recirculation is presented as a broadband noise, therefore having well-separated RCs that decompose the oscillations is not of interest when the entire effect of inlet recirculation is to be isolated. In this study, a tailored performance analysis has been conducted to identify which will be the best window length for RC2 and RC9, as were found to be good candidates for the isolation characterisation of inlet recirculation as shown in Figure 3

The potential for the isolation of the inlet recirculation by the use of SSA-technique had been established in previous sections. Further investigation of the parameters governing the performance of SSA and the proposition of the basis of a monitoring system was now necessary. RC 2 had been established as a offering a strong potential for the basis of condition monitoring of the instability of the system, emphasised through examination of Fig $6 \mathrm{~b}$ The resolution of the SSA decomposition was known to be governed by the window length. The effect of window length modification on RC 2 was, therefore, to be now considered. The 
RMS of the extracted component was used as an indicator of the instability of the flow, with a maximum magnitude at $\mathrm{TOA}=22 \%$, the point at which the instability representative of the inlet recirculation was most prominent. This was to be compared to a stable flow regime at TOA $=30 \%$. These choices can be understood by reference to Fig. 6b The performance of various window lengths were to compared as presented in Fig. 7a, where window length was plotted against the relative difference between reference and recirculation conditions, thereby demonstrating the variation in the ability of $\mathrm{RC} 2$ to indicate recirculation.

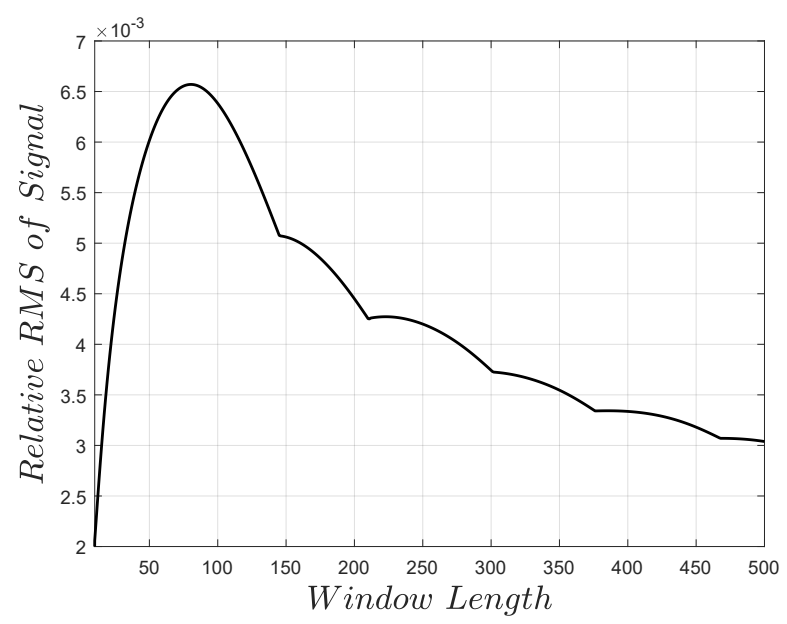

(a) Based on RC 2

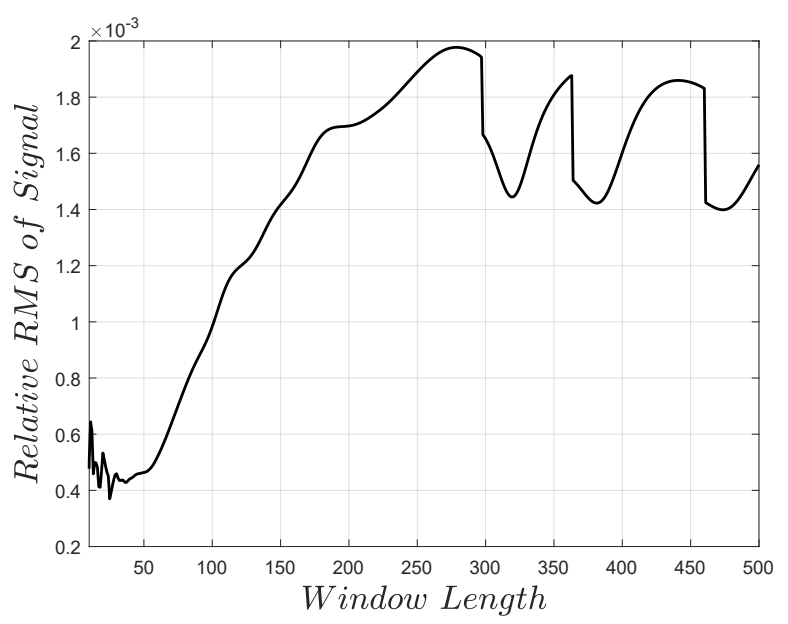

(b) Based on RC 9

Figure 7: Window length optimisation

The optimal window length for the clearest possible diagnosis of inlet recirculation by inspection of RC 2 could be obtained from inspection of Fig. 7a It appeared that the point at which there was greatest difference between reference and recirculation conditions was at approximately $L=80$. This was comparable to the window length of 50 used to generate the promising spectral maps in Fig. 5 and 6 and selected in previous studies on this test rig [41, 42. This also confirms the validity of a known practice for the SVD method, where a window length is chosen approximate to the blade passing frequency [32]. The performance of this window length was not unexpected from examination of parallel plots of frequency spectra, where at $L=10, \mathrm{RC} 1$ almost entirely dominated the decomposition. This was similar to the effect of an inadequate window length presented by Komatsubara et al. [33. On the other side at $L=200$ the recirculation was captured by a broad range of higher components as shown in Figure $3 \mathrm{c}$

It would seem that a monitoring system could be based on these choices of window length and RC, but it must be remembered that the nature of the decomposition changed with window length, and that RC 2 will not continue to be the best choice of component at higher values of $L$. This can be understood by reference to Fig. [3c, where various RCs were plotted at a window length of 200. In this plot it was clear that RC 2 does not capture frequencies in the range of the recirculation and that a better choice of component for the 
basis of a monitoring system would be RC 9, for example. It was, therefore, thought necessary to investigate the possibility of RC 9 as a basis for a control system, first by obtaining an optimal window length for this RC, following an identical process as for RC 2. Fig. 7b captured the results of this process.

As was expected from interpretation of Fig. 3c the optimal window length markedly increased at this higher choice of component. The point at which the difference between reference and the recirculation conditions appeared to increase up to approximately $L=250$, after which the trend degenerated, likely a result of the increased susceptibility to noise of this higher component. It should be noted that this was similar to the window length of 200 plotted in Fig. 3c at to be expected considering that RC 9 was chosen on the basis of this plot. This result suggested some potential of higher RCs for inlet recirculation identification, but with the requirement of an increased window length. It should be noted that these window lengths were selected on the basis of TOA $=22 \%$ as indicative of the recirculation and it was to be ensured that any potential indicator would be capable of detecting the onset of the recirculation at TOA $=26 \%$, such that the associated drop in compressor pressure ratio indicated in [41] could be avoided.

\subsubsection{Study on a potential monitoring system}

After the demonstration on how each of the proposed window length and RC choices would behave with variation in throttle position, thus clearly outlining the options available for a potential monitoring system. Two options were established in section 4.3.1 The less computationally demanding window length of $L=80$ or the higher resolution decomposition at $L=250$, with each choice made according to a proposed RC selection of 2 or 9 , respectively. In order to enhance understanding of the function of SSA and to outline the effect of the parameters available to the researcher, RC and $L$, the RMS values of RCs 1,2 and 9 were plotted at both window length options. Fig. 8 compares these RC choices at the optimal window lengths obtained in section 4.3 .

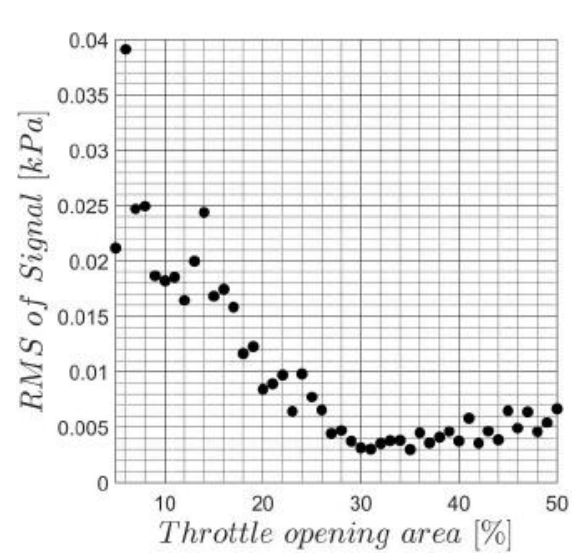

(a) Using only $\mathrm{RC}=1$

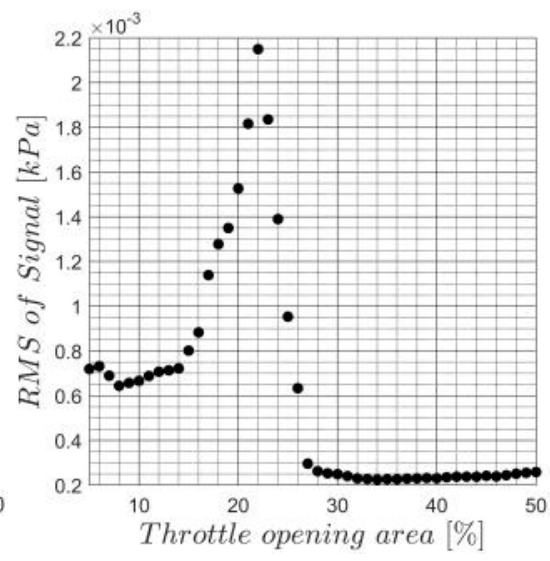

(b) Using only $\mathrm{RC}=2$

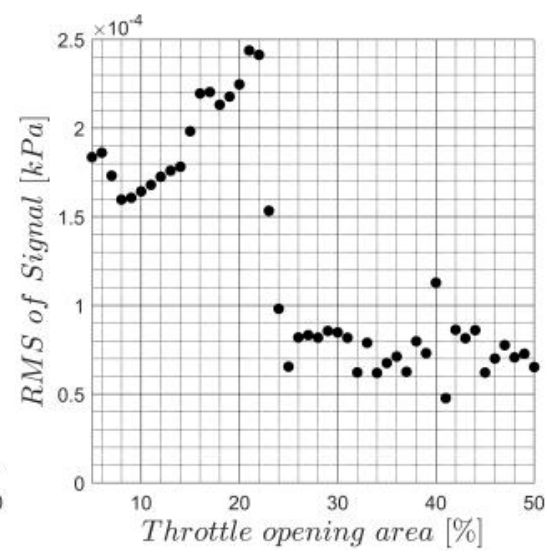

(c) Using only $\mathrm{RC}=9$

Figure 8: RMS variation of pressure signals for different TOA measured at $\mathrm{p}_{\mathrm{s}-\mathrm{imp} 1}, L=80,5 \%<\mathrm{TOA}<50 \%$ 
Fig. 8 allows the visualisation of a potential monitoring method to be demonstrated, as well as elucidation of the effect of parameter choice. Fig. 8a demonstrated the basic capability of SSA. It was clearly seen that a maximum value of RMS could be derived from stable conditions and the recirculation diagnosed in excess of this limit. With RC 2 it is possible to detect the recirculation at TOA $=26 \%$ by a very strong and clear increase in RMS. This signal was also able to give indication of the transition from the inlet recirculation to subsequent transient instability conditions. This plot clearly indicated that the inlet recirculation was most prominent at $\mathrm{TOA}=22 \%$, providing justification for the choice of this throttle position to calibrate window length selection in section 4.3.1. At this window length, RC 2 appears to be effectively dedicated to representation of the inlet recirculation and does not capture any other significant flow characteristic. RC 9 was not an ideal choice of diagnostic component at this window length. The greater influence of noise on this higher, lower magnitude component, can clearly be seen, particularly in the region of stable flow. RC 9 fails to give any detection of the recirculation until $\mathrm{TOA}=23 \%$, near the point at which this phenomenon is most prominent. If RC 9 is to be used, a higher window length of must be chosen.

Fig. 9 compares the relative values of RMS of RCs 1,2 and 9 at such a window length, $L=250$. As was expected, RC 9 now produced more promising results. The plots seemed apparently reversed between Figs. 8 and 9 with notable resemblance between Figs. $8 \mathrm{a}$ and $9 \mathrm{~b}$ RC 9 is now operating at its optimal window length, and detects the inlet recirculation at $\mathrm{TOA}=26 \%$. RC 9 captures the inlet recirculation with greater clarity than RC 2 at $L=80$, with the transient instability and the deep surge influencing the RMS value to a lesser extent outwith the region of recirculation. These features continue to be captured by lower RCs, primarily $\mathrm{RC} 1$, at higher window lengths. $\mathrm{RC} 2$ at $L=250$ appeared initially to bear resemblance to $\mathrm{RC}$ 9 at $L=80$, but in this case RC 2 remained able to capture the recirculation at TOA $=26 \%$. This was a critical outcome of the study, as it was now apparent that the selection of a higher window length did not prevent the detection of the inlet recirculation by RC 2, despite the use of this component being non-optimal at this $L$ value. In this case it can be confirmed that $\mathrm{RC} 2$ is a robust independent component that contains features characteristic of inlet recirculation. 


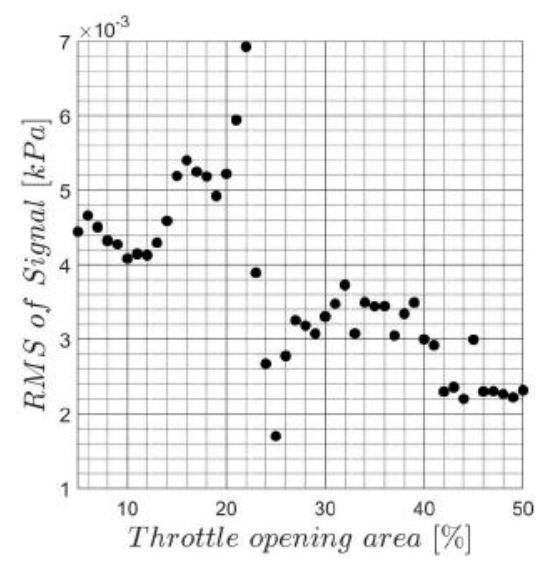

(a) Using only $\mathrm{RC}=1$

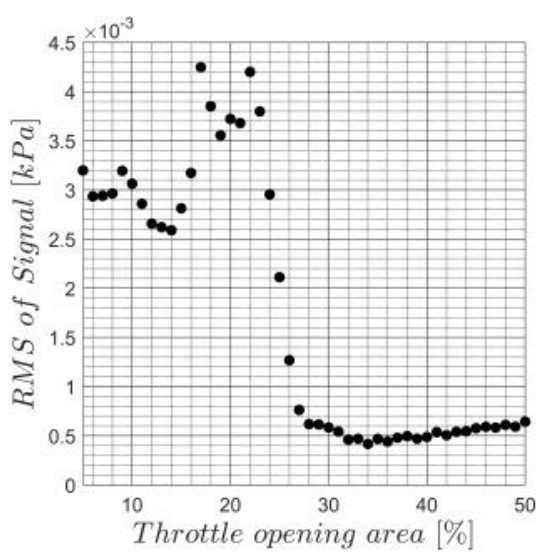

(b) Using only $\mathrm{RC}=2$

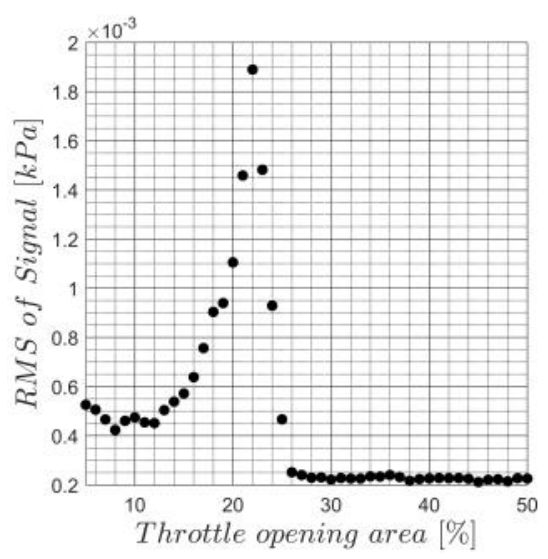

(c) Using only $\mathrm{RC}=9$

Figure 9: RMS variation of pressure signals for different TOA measured at $\mathrm{p}_{\mathrm{s}-\mathrm{imp} 1}, \mathrm{~L}=250,5 \%<\mathrm{TOA}<50 \%$

If the increased computational demand was considered, there appeared to be no real justification for adopting the more refined technique wherein window length was increased and a higher RC selected. This is because of the wide band of frequencies induced by inlet recirculation. SSA is capable of extracting specific frequencies with a suitably high window length. In this case, where the phenomenon can be extracted from a broad section of the spectrum, it is more efficient to choose a lower, less demanding $L$ value and simply select RC2, thereby discarding all frequencies below the region of interest, as was seen in the spectral maps in section 4.2 If, for example, it was desired to purely extract the blade passing frequency, a high window length would have to be set, and the associated RC identified, but in the case of the inlet recirculation the parameters chosen in order to generate the plots in Fig. $8 \mathrm{a}$ would be sufficient.

\subsection{Advantage of protection \& control approach}

The classic anti-surge systems impose a 10\% - 15\% range limitation compared to the predicted surge margin [49]. The surge limit usually calculated based on theoretical predictions [11, 50. Hence there is a lot of uncertainty about the behaviour of such a system, especially with a change of external conditions, machine wear or heat transfer effects coming from another elements of the system [51]. The choice of such a big surge margin allows to compensate potential appearance of additional effects that might affect the surge limit change.

Presented system is of type 'protection \& control' as described by [9] and in the section 1 It reacts based on the condition of the machine registered in real-time. Presented isolation of the component containing the inlet recirculation allows to get clear warning signal at TOA $=26 \%$, which is $9 \%$ prior to the transient instability and $15 \%$ prior to the deep surge. This gives robust information about the surge proximity and provides much space for potential reaction.

It has to be outlined that the surge margin does not need to also protect the machine from the inlet recirculation. This phenomenon is not causing critical risk for the system operation and it can continue 
working by the cost of gradual efficiency loss [19]. Nevertheless, clear inlet recirculation detection allows one to be aware of the surge proximity. Knowing where the surge is, the machine operator can decrease the surge margin and let the machine approach it by the range of $3 \%-5 \%$. The operator might also decide to protect the machine from all instabilities including the transient instability and the inlet recirculation in situations where compression efficiency is more important than the wide working range.

The practical application of this method, therefore, would depend on the use scenario of the compressor. In all situations the advantage of the 'protection \& control' approach is clear - decision is made based on the real indication of much higher accuracy and relevance rather than on theoretical limits. Real-time indication includes all additional effects of surge limit shifting connected with the machine working conditions, wear, heat transfer effects etc.

The efficiency of such a system could be also tested in different impeller speeds to prove its universality. It is known that the spectral structure of certain instabilities are influenced by this parameter. It has to be pointed out, however, that recent studies have shown that the inlet recirculation onset point also changes with the impeller speed and always precedes the surge by a similar distance along the performance curve 52. This means that the protection \& control systems based on inlet recirculation are indeed reacting prior to the surge for a wide range of the speed lines. This matter could addressed to further studies with the SSA method.

\subsection{Advantage of SSA for compressor monitoring}

The use of SSA allows one to get the clear indication of surge proximity. Analysis of particular flow phenomena is a good way to deliver direct information about their appearance. This study has shown that the inlet recirculation can be easily isolated from the signal and provide very sharp information regarding their appearance. This makes the SSA a good candidate for 'protection \& control' anti-surge system. The other advantage of this method is that it can be easily implemented and quickly calculated based on the raw signal without application of additional filters. The disadvantage of this method lies in the fact, that the proper component needs to be chosen for monitoring. Further study should be addressed to see which component provides best monitoring possibilities on different machines.

This class of methods is more suitable for anti-surge systems than the CWT. The wavelet analysis is good for research purpose and provides information on the spectral structure of the flow instability. The use of this method, however, in surge protection is not widely applied, as it does not provide clear and amplified indication of instability onset. [28].

\section{Conclusions}

The aim of this work was to investigate a potential methodology for centrifugal compressor's monitoring to ensure safe working conditions and eventually promote high performance. The potential use of singular spectrum analysis as a tool for diagnosis of instability in a centrifugal compressor was examined in detail. The 
ability of SSA to decompose a complex signal was represented by parallel plots of the frequency spectra of the various components generated by SSA. These components were inspected in order to obtain understanding of the potential of SSA to extract particular flow features. It was seen that inlet recirculation, an early indicator of instability preceding surge, could potentially be isolated from the complex signals obtained upstream of the compressor impeller. Spectral maps were generated, according to a technique present in existing literature wherein the full signal was analysed to be compared with discomposed fractions of the signal obtained by SSA. This suggested a monitor system may be developed based on analysis of a particular component. The governing parameters of this proposed monitoring system were investigated, namely the choice of independent RCs and the Window Length, the metric by which the resolution of the decomposition was determined. It was found that the optimal window length depended on the choice of component. Two potential combinations of these parameters were investigated in order to enhance understanding of the process of decomposition. The function of these options in a potential monitor system were demonstrated by analysis of their behaviour with variation in throttle position. In the case of inlet recirculation, it was suggested that a relatively low window length may be adopted and the second component used to identify inlet recirculation. These parameter choices were able to capture the onset of this instability and, therefore, could be used to provide the basis of a method by which operation in the region of recirculation could be avoided as an early indicator of instability.

\section{Acknowledgments}

This work was funded by the Polish National Centre for Research and Development (Grant No. Lider/447/L6/14/NCBR/2015).

\section{References}

[1] H. P. Bloch, A Practical Guide to Compressor Technology, Wiley-Interscience, Hoboken, New Jersey, 2006.

[2] J. T. Gravdahl, O. Egeland, Compressor Surge and Stall Modelling and Control, Springer, London, 1999.

[3] B. D. Jager, Rotating stall and surge control: a survey, Proceedings 34th. IEEE Conference on Decision and Control 2 (1995) 1857-1862.

[4] H. W. Emmons, C. E. Pearson, H. P. Grant, Compressor surge and stall propagation, Transactions of the ASME 77 (4) (1955) 455-469.

[5] E. M. Greitzer, Surge and rotating stall in axial flow compressors, part i: Theoretical compression system model, Jo. Eng. Power 98 (2) (1976) 190-198. 
[6] K. E. Hansen, P. Jorgensen, P. S. Larsen, Experimental and theoretical study of surge in a small centrifugal compressor, J. Fluids Eng. 107 (1981) 391-395.

[7] K. Kabalyk, W. Kryowicz, G. Likiewicz, L. Horodko, R. Magiera, Experimental investigation of the influence of the inlet duct configuration on the unstable operation of a single-stage centrifugal blower,

[17] S. Mizuki, Y. Oosawa, Unsteady flow within centrifugal compressor channels under rotating stall and surge, J. Turbomach. 114 (2) (1992) 312-320.

[18] H. Tamaki, Experimental study on surge inception in a centrifugal compressor, J. Fluid Mach. Syst. 2 (4) (2009) 409-417.

[8] J. Galindo, A. Tiseira, R. Navarro, D. Tar, C. Meano, Effect of the inlet geometry on performance, surge margin and noise emission of an automotive turbocharger compressor, Applied Thermal Engineering $110(2017) 875-882$.

[9] K. K. Botros, J. Henderson, Developments in centrifugal compressor surge control a technology assessment, J. Turbomach. 116 (2) (1994) 240-249.

[10] D. A. Fink, N. A. Cumpsty, E. M. Greitzer, Surge dynamics in a free-spool centrifugal compressor system, J. Turbomach. 114 (2) (1992) 321-332.

[11] J. Galindo, A. Tiseira, F. J. Arnau, R. Lang, On-engine measurement of turbocharger surge limit, Experimental Techniques 37 (1) (2013) 47-54.

[12] A. H. Epstein, J. E. Ffowcs Williams, E. M. Greitzer, Active suppression of aerodynamic instabilities in turbomachines, J. Propul. Power 5 (2) (1989) 204-211.

[13] F. E. Pinsley, G. R. Guenette, A. H. Epstein, E. M. Greitzer, Active stabilization of centrifugal compressor surge, J. Turbomach. 113 (4) (1991) 723-732.

[14] J. T. Gravdahl, Modeling and control of surge and rotating stall in compressors, Ph.D. thesis, Norwegian University of Science and Technology (1998).

[15] J. S. Simon, L. Valavani, A. H. Epstein, E. M. Greitzer, Evaluation of approaches to active compressor surge stabilization, J. Turbomach. 115 (1993) 57-67.

[16] G. Likiewicz, et al., Identification of phenomena preceding blower surge by means of pressure spectral maps, Exp. Therm. Fluid. Sci. 54 (2014) 267-278.

9] C. Schreiber, Inlet recirculation in radial compressors, Ph.D. thesis, University of Cambridge (2018). 
[20] G. Liśkiewicz, K. Sobczak, M. Stickland, W. Kryłłowicz, Numerical study of off-design centrifugal compressor operation and flow phenomena preceding surge, in: Turbo Expo: Power for Land, Sea, and Air, Vol. 50992, American Society of Mechanical Engineers, 2018, p. V02AT45A035.

[21] R. J. Mckee, C. E. Edlund, Method and apparatus for detecting the occurrence of surge in a centrifugal compressor, uS Patent 6,981,838 (Jan. 3 2006).

[22] J. Yin, Centrifugal compressor with surge control, and associated method, uS Patent 8,272,832 (Sep. 25 2012).

[23] S. Mizuki, Y. Oosawa, Unsteady flow within centrifugal compressor channels under rotating stall and surge.

[24] N. Bulot, X. Ottavy, I. Trebinjac, Unsteady pressure measurements in a high-speed centrifugal compressor, Journal of Thermal Science 19 (1) (2010) 34-41.

[25] P. Lawless, S. Fleeter, Rotating stall acoustic signature in a low-speed centrifugal compressor: Part 1 vaneless diffuser.

[26] T. Turunen-Saaresti, J. Larjola, Unsteady pressure field in a vaneless diffuser of a centrifugal compressor: an experimental and computational analysis, Journal of Thermal Science 13 (4) (2004) 302-309.

[27] L. Horodko, Identification of rotating pressure waves in a centrifugal compressor diffuser by means of the wavelet cross-correlation, International Journal of Wavelets, Multiresolution and Information Processing 4 (02) (2006) 373-382.

[28] G. Likiewicz, L. Horodko, Time-frequency analysis of the surge onset in the centrifugal blower, Open Eng. 5 (1) (2015) 299-306.

[29] N. Buffaz, I. Trébinjac, Aerodynamic instabilities in transonic centrifugal compressor, Mechanics \& Industry 15 (3) (2014) 191-196.

[30] A. Mertins, D. A. Mertins, Signal analysis: wavelets, filter banks, time-frequency transforms and applications, John Wiley \& Sons, Inc., 1999.

[31] M. Stajuda, G. Liśkiewicz, D. Garcia, Flow instabilities detection in centrifugal blower using empirical mode decomposition, Proceedings of Global power and propulsion society, Beijing Conference 2019.

[32] C. Gu, K. Yamaguchi, T. Nagashima, H. Higashimori, Observation of centrifugal compressor stall and surge in phase portraits of pressure time traces at impeller and diffuser wall, Journal of fluids engineering 129 (6) (2007) 773-779.

[33] Y. Komatsubara, S. Mizuki, Dynamical system analysis of unsteady phenomena in centrifugal compressor, Journal of Thermal Science 6 (1) (1997) 14-20. 
[34] N. Golyandina, V. Nekrutkin, A. Zhigiljavsky, Analysis of Time Series Structures: SSA and Related Techniques, Chapman \& Hall CRC, Boca Raton, Florida, 2001.

[35] S. Polukoshko, G. Hilkevica, V. Gonca, Nonstationary processes studying based on caterpillar ssa method, Mech. Mach. Theory 23 (2015) 999-1008.

[36] M. Ghil, et al., Advanced spectral methods for climatic time series, Rev. Geophys. 40 (1) (2002) 3-41.

[37] I. Jalón-Rojas, S. Schmidt, A. Sottolichio, Evaluation of spectral methods for high-frequency multiannual time series in coastal transitional waters: advantages of combined analyses, Limnology and Oceanography: Methods 14 (6) (2016) 381-396.

[41] D. Garcia, M. Stickland, G. Likiewicz, Dynamical system analysis of unstable flow phenomena in centrifugal blower, Open Eng. 5 (1) (2015) 232-342.

[42] D. Garcia, G. Likiewicz, Stable or not stable? recognizing surge based on the pressure signal, Trans. Ins. Fluid-Flow Mach. 133 (2016) 55-68.

[43] F. J. Alonso, J. M. Del Castillo, P. Pintado, Application of singular spectrum analysis to the smoothing of raw kinematic signals, J. Biomech. 38 (2005) 1085-1092.

[44] B. De Jager, Rotating stall and surge control: A survey, in: Proceedings of 1995 34th IEEE Conference on Decision and Control, Vol. 2, IEEE, 1995, pp. 1857-1862.

[45] G. Tzagkarakis, M. Papadopouli, P. Tsakalides, Trend forecasting based on singular spectrum analysis of traffic workload in a large-scale wireless lan, Performance Evaluation 66 (3-5) (2009) 173-190.

[46] C.-H. Loh, C.-H. Chen, T.-Y. Hsu, Application of advanced statistical methods for extracting long-term trends in static monitoring data from an arch dam, Structural Health Monitoring 10 (6) (2011) 587-601.

[47] M. A. de Oliveira, J. Vieira Filho, V. Lopes Jr, D. J. Inman, A new approach for structural damage detection exploring the singular spectrum analysis, Journal of Intelligent Material Systems and Structures 28 (9) (2017) 1160-1174.

[48] H. Hassani, Singular spectrum analysis: methodology and comparison (2007) 1160-1174. 
[49] R. Van den Braembussche, Design and Analysis of Centrifugal Compressors, John Wiley \& Sons, 2019.

[50] S. Antas, An anlytical and numerical method of calculating the surge margin of compressor of turboshaft engine with free power turbine., Archive of Mechanical Engineering 48 (4) (2001) 373-397.

[51] J. Larjola, Simulation of surge margin changes due to heat transfer effects in gas turbine transients, in: Turbo Expo: Power for Land, Sea, and Air, Vol. 79474, American Society of Mechanical Engineers, 1984, p. V002T04A003.

[52] G. Liśkiewicz, K. Kabalyk, A. Jaeschke, F. Grapow, M. Kulak, M. Stajuda, W. Kryłłowicz, Unstable flow structures present at different rotational velocities of the centrifugal compressor, Energies 13 (16) (2020) 4146. 